\title{
A View of Supernova Remnant CTB 37A with the Fermi Gamma-ray Space Telescope
}

\author{
T. J. Brandt ${ }^{1, *}$ \\ 9, av du Colonel Roche BP 44346 / 31028 Toulouse Cedex 4 \\ on behalf of the Fermi Large Area Telescope Collaboration
}

\begin{abstract}
Supernovae and their remnants have long been favored as cosmic ray accelerators. Recent data from the Fermi Gamma-ray Space Telescope has given us an improved window into such sources, including the remnant CTB 37A. Using the Fermi Large Area Telescope, we found significant gammaray emission coincident with the remnant, which also emits in radio, X-ray, and very high energy gamma-rays. We modeled the multiwavelength spectrum using a combination of hadronic and leptonic emission with reasonable parameter values and determined that CTB 37A is a potential cosmic ray accelerator commensurate with direct observations. By assembling statistically significant populations of such objects, we will be able to more fully illuminate the mystery of cosmic ray origins.
\end{abstract}

Keywords: CTB 37A; Supernova Remnants; Gamma-rays; Cosmic rays

\section{Introduction}

The mystery of the origin of cosmic rays (CRs) has lasted for nearly 100 years. Energetics arguments strongly suggest supernovae and their remnants (SNe \& SNRs) may be a source of Galactic CRs. By observing such potential CR accelerators in multiple wavelengths, we can constrain their particle

\footnotetext{
${ }^{*}$ Corresponding author

Email address: brandt@cesr.fr (T. J. Brandt)

${ }^{1}$ Université de Toulouse, UPS-OMP, IRAP and CNRS, IRAP
} 
populations, the acceleration processes they undergo, and thus the sources' ability to accelerate CRs up to some of the highest energies observed.

We do so for the SNR CTB 37A, which we detect with the Fermi Gammaray Space Telescope (Fermi). The slightly extended emission coincides with the nominal position of CTB 37A, existing radio and X-ray data, and H.E.S.S. very high energy gamma-ray observations. Previous studies suggest that the SNR may be interacting with molecular clouds seen in CO to be positionally coincident with the remnant (Reynoso and Mangum, 2000). The clouds are also kinematically associated with several $\mathrm{OH} 1720 \mathrm{MHz}$ masers, easily explained through collisional shock excitation (Frail et al., 1996). In this way, SNR-cloud interactions may be another source of both CRs and gamma-rays emitted by CTB 37A. Motivated by these observations, we present a model of the multiwavelength spectrum which both reasonably reproduces the Fermi data and suggests that this SNR could be accelerating CRs up to the observed energies. An independent analysis of CTB 37A (Castro and Slane, 2010) reaches similar conclusions.

\section{Fermi LAT Analysis: Methods and Results}

The Fermi Gamma-ray Space Telescope, launched 11 June 2008, contains the Gamma-ray Burst Monitor and the Large Area Telescope (LAT). The LAT's silicon tracker modules and Cesium Iodide calorimeter are sensitive to photons in a broad energy range $(\sim 0.02$ to $>300 \mathrm{GeV})$ with a large effective area $\left(\sim 8,000 \mathrm{~cm}^{2}\right.$ for $E>1 \mathrm{GeV}$ for on-axis events) over a large field of view $(\sim 2.4 \mathrm{sr})$. The front (first 12) tracker planes have thin Tungsten converter foils enabling a smaller point spread function (PSF) while the back (last 4) planes' thicker converters permit a larger effective area.

We used approximately 18 months of the standard, low background diffuse class LAT data, collected from 8 Aug 2008 to 12 Feb 2010. A zenith cut of $105^{\circ}$ minimizes Earth albedo gamma-rays while limiting the energy range to $0.2<E<50 \mathrm{GeV}$ minimizes the systematic error. We used the standard science tools (v9r15p6) and instrument response functions (IRFs) (P6_V3) ${ }^{2}$ to fit a $4.5^{\circ}$ region of interest (ROI) around the known location of the source (Green, 2009). In particular, we used the gtlike binned maximum likelihood fit to optimize the spectral parameters of all sources from the 1FGL catalog

\footnotetext{
${ }^{2}$ Available at the Fermi Science Support Center. http://fermi.gsfc.nasa.gov/ssc
} 
(Abdo et al., 2010a) in the ROI, iterating to include only significant sources $(>4 \sigma)$. As CTB 37A is quite near the Galactic plane, the smaller ROI limited the number of sources included in the gtlike fit of the region to a more manageable number while still containing a sufficient number of PSF length scales. We also included the standard isotropic ${ }^{3}$ and Galactic diffuse models: isotropic_iem_v02 and gll_iem_v02, respectively. Further details of the LAT instrument and data reduction may be found in Atwood et al. (2009).

\subsection{Detection}

This analysis yielded an $18.6 \sigma$ detection of a point source coincident with the nominal CTB 37A position, as seen in Figure 1, a map of residual FermiLAT emission after subtracting all the nearby, fit sources and diffuse backgrounds. For this detection, we used gtlike to quantify the spectral shape with a power law. Discussion of the fit with another reasonable spectral form, the exponentially cutoff power law, may be found in 2.4. In Section 3 we explore a physically motivated model.

\subsection{Diffuse Model}

Since CTB 37A lies just above the Galactic plane we ensured that the particular Galactic diffuse background employed was not biasing our detection by examining the global likelihood of three different diffuse models including several variations for the two most reasonable models. As expected, the DIRBE $60 \mu \mathrm{m}$ infrared map, mainly tracing thermal emission from e.g., dust heated by stars, including interplanetary dust heated by the Sun, poorly represents the diffuse Galactic gamma-ray background and so led to a low global fit likelihood relative to the standard model. Using a variation on the standard model, fitting it just to a region around the Galactic center $\left((l, b)= \pm\left(20^{\circ}, 20^{\circ}\right)\right)$, also did not substantially improve the global fit to the ROI. Neither including a faint pulsar in the region found after the 1FGL catalog was published nor modulating the original Galactic diffuse model by a power law spectral shape significantly improved the global fit, particularly considering the addition of extra parameters in each case.

The global fit did improve when using a variation of the Galactic diffuse model with finer spatial resolution based on 19 months' data. The fit for

\footnotetext{
${ }^{3}$ All contributions whose spatial distribution is assumed to be isotropic: the extragalactic background, unresolved sources, and the instrumental background.
} 


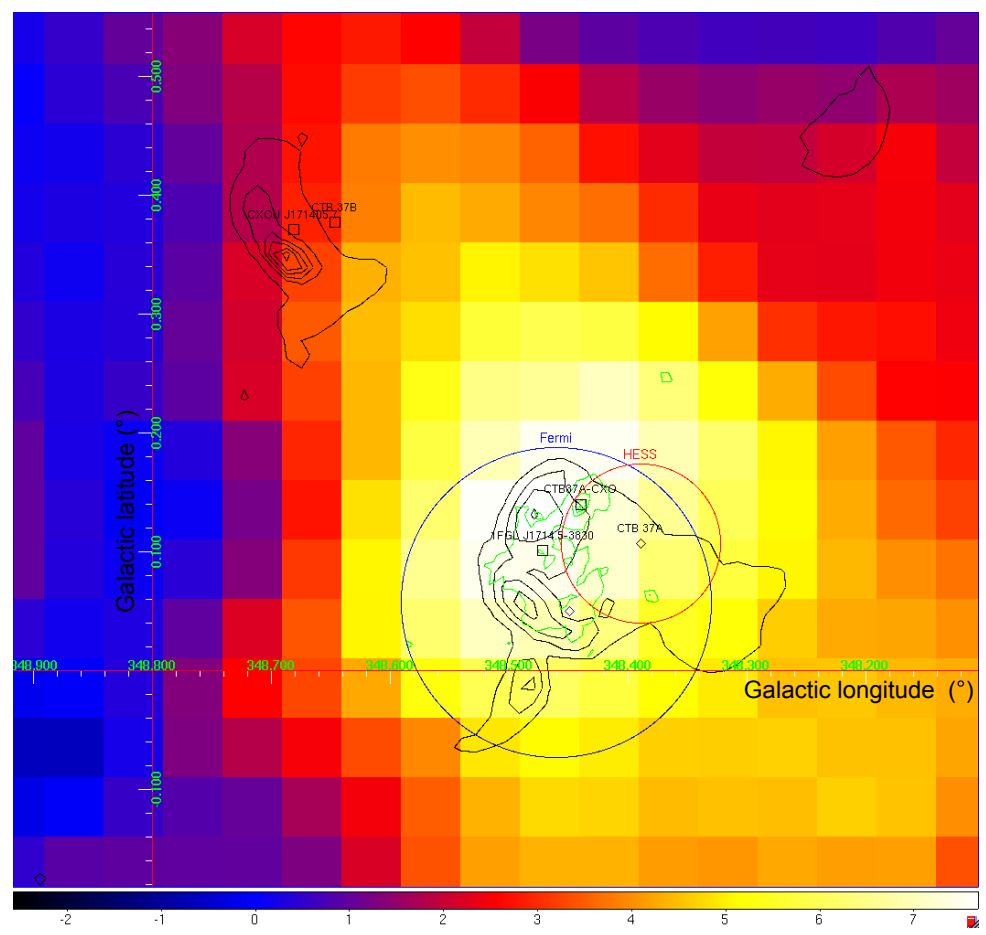

Figure 1: The Fermi-LAT residual map (color scale) in the energy range $0.2-50 \mathrm{GeV}$ shows an excess clearly associated with both the nominal position (black diamond (Green, 2009)) and radio emission (southern black contours (Kassim et al., 1991)) of CTB 37A and not CTB 37B (northern black contours). The Fermi-LAT emission's most likely position and Gaussian extension (blue circle) also coincides with the H.E.S.S. source (red circle (Aharonian et al., 2008)) as well as the XMM-Newton MOS1 detector X-ray data (green contours) in the energy range $0.2-10 \mathrm{keV}$. We performed a blind search for pulsations at the four positions indicated with black squares: at the 1FGL catalog position and the nearby possible X-ray source for CTB $37 \mathrm{~A}$ and at the nominal and coincident X-ray source positions for CTB 37B. 
this model further improved as we included sources from the 1FGL catalog which had a lower significance $(>3 \sigma)$ in the initial fit and as we modulated the Galactic diffuse model by a power law in energy. Combining these two variations and further including in the combination the faint pulsar both yielded moderate improvements in the global likelihood over any one variation singly. While the 19 months' model variations yielded the best global fit, they remain under development, and the other models are either worse or show negligible improvement in the global fit relative to the standard diffuse model. Further, none of the reasonable models significantly influence the results for the point source at the nominal CTB 37A position. The minimum source significance was within $8 \%$ of the average of all reasonable variations and the best-fit power law index remained within $3 \%$ of the average. We therefore conclude that the standard Galactic diffuse model used in the analysis is adequate to the task and further that neither it nor its variations greatly affect our results.

\subsection{Location and Extension}

Having determined the existence of a significant source in the vicinity of the CTB 37 complex, we simultaneously fit for the most likely position and extension of the source. To do so, we stepped around a spatial grid to determine the most likely position for a given (Gaussian) extension, recentering the grid as needed to more completely included the largest extensions $\left(0.3^{\circ}-0.5^{\circ}\right)$. We interpolated the most probable extension bounded by a point source and $0.5^{\circ}$ and then iterated over a finer range of extensions on a finer grid spacing (from $1.0^{\circ}$ to $0.1^{\circ}$ on a $5 \times 5$ grid) to improve the final location, extension, and their respective statistical errors. The final location is that associated with the most likely extension. This method was previously employed in e.g. Abdo et al. (2010c). We also verified the results using the alternate tool Sourcelike (Abdo et al., 2010d, e.g.).We obtained an identical extension as for the grid method, and further determined that a uniform disk was no more likely than a Gaussian extension.

We thus found a position for the Fermi-LAT source of $\mathrm{RA}=258.68^{\circ} \pm$ $0.05_{\text {stat }}^{\circ} \pm 0.006_{\text {sys }}^{\circ}, \mathrm{DEC}=-38.54^{\circ} \pm 0.04_{\text {stat }}^{\circ} \pm 0.02_{\text {sys }}^{\circ}$. The symmetrical Gaussian with an extension of $\sigma=0.13^{\circ} \pm 0.02_{\text {stat }}^{\circ} \pm 0.06_{\text {sys }}^{\circ}$ was most likely, with a significance of $\sim 4.5 \sigma$. The position and extension can be seen in Figure 1 overlaid on the Fermi-LAT residual map, along with the radio contours for CTB 37A and B and the position and extension of the H.E.S.S. source coincident with CTB 37A. We clearly see that the Fermi-LAT source 
is coincident with both the CTB 37A radio contours and the H.E.S.S. source, and further that it is inconsistent with CTB 37B.

We further checked that the Galactic diffuse model and possible nearby sources did not affect our extension and location determination by performing the same analysis with three of the most reasonable models' variations: including the faint pulsar in the standard model, including less-significant sources from the 1FGL catalog in the 19 months' diffuse model variation, and including the less-significant sources and modulating the 19 months variation by a power law in energy. In all three cases, the position and extension found were within statistical errors of the originally determined position and extension. Thus we conservatively take the extreme difference as the systematic error due to the diffuse model, which is only $\sim 30 \%$ of the extension and well below the statistical error $\left(< \pm 0.015^{\circ}\right)$ for the position. The diffuse model systematic error thus derived is smaller than the PSF.

We also examined the extension and localization using subsets of events with a nominally better PSF: those at higher energy $(2-50 \mathrm{GeV})$ and those photons which convert in the front of the tracker, where the foils are thinnest and thus cause the least scatter. In both cases, the position remained stable and the extension within the statistical errors. We again use the maximum difference to estimate the order of magnitude of systematic error from the PSF, which we combined in quadrature with the systematic error from the diffuse model.

\subsection{Variability and Pulsations}

Fermi GST has identified emission from over 70 pulsars and variable emission from over 180 blazars. As pulsing or variable emission can strongly influence the results found for a steady source, we performed two searches of the Fermi-LAT source coincident with the CTB 37 complex: a search for long-term variability in the light curve and a blind pulsation search of the most likely candidates in the region. We found no significant variability in the 2-week binned light curves when fitting the spectrum with either a power law or a power law with exponential cutoff (PLEC) spectral shape. All fluxes remained within error of $1 \sigma$ of the average except for the two lowest, whose errors are systematically underestimated due to low statistics, and the third highest flux, which had an unusually small error. Replacing these with the errors determined for their nearest neighbors (in flux) yields a reduced $\chi^{2}$ compared to a constant, average flux of $\chi^{2} /$ d.o.f. $\approx 1.4$ and 5.7 for the power law and PLEC, respectively. Thus, we do not observe long-term variability. 
To check for pulsar-like emission, we performed a blind pulsation search at four likely locations coincident with the CTB 37 complex, seen as squares in Figure 1, using 18 months diffuse events with $E \geq 300 \mathrm{MeV}$ in a ROI of $r \leq 0.8^{\circ}$. We employed a differencing method with a maximum frequency of $64 \mathrm{~Hz}$, a maximum coherency window of 524, 288 seconds, and a spin down frequency $(\dot{F})$ ranging from 0 to $3.86 \times 10^{-10}$, equal to that of the Crab. The 1FGL catalog source J1714.5-3830, coincident with the Fermi-LAT source and CTB 37A radio contours, and the X-ray source CXOJ171419.8m383023, coincident with the Fermi-LAT, radio, and H.E.S.S. CTB 37A sources, showed no pulsations. We also examined the nominal position of CTB 37B (RA $=258.49, \mathrm{DEC}=-38.20)$ and the nearby X-ray source CXOU_J171405.7, more coincident with CTB 37B's radio contours, for pulsations, likewise finding none. Further details on the Fermi-LAT blind pulsation search may be found in Dormody et al. (2011).

We also find the Fermi-LAT source coincident with CTB 37A inconsistent with a pulsar hypothesis as the best-fit spectral shape, determined with gtlike to be a PLEC, while consistent with the standard pulsar one, shows only a $\sim 1.5 \sigma$ improvement over the simple power law while adding an extra degree of freedom. Further, the best fit cutoff energy $\mathrm{E}_{\text {cut }}=28.2_{-10.5-21.6}^{+29.8+67.8} \mathrm{GeV}$ is both an order of magnitude higher than typical pulsar cutoff energies (e.g. Abdo et al. (2010b)) and moreover is also poorly fit, as evidenced by the large errors which are consistent with no cutoff detection. Thus, we find it unlikely that the detected emission comes from a pulsar.

\subsection{Spectrum}

To create the Fermi-LAT spectral points shown in Figure 2, we used $g t$ like to fit power laws to the data within each of 15 logarithmically-spaced energy bins. The narrowness of the bins in energy space ensures that the exact functional form and parameters of the power law do not substantially influence the values of the points themselves. We do not show the lowest $(0.2-0.3 \mathrm{GeV})$ and highest $(30-50 \mathrm{GeV})$ energy bins due to insufficient statistics. The variations in the Fermi-LAT data points are not overwhelmingly significant in light of systematic errors for this standard Fermi-LAT analysis. 


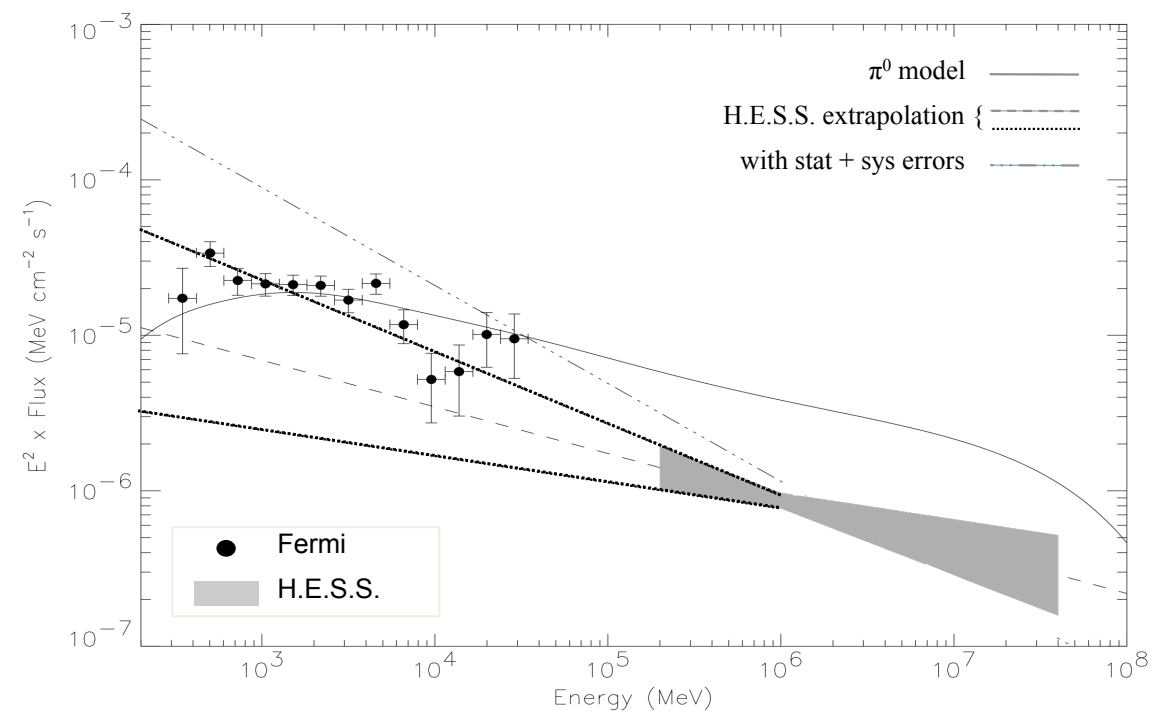

Figure 2: The Fermi-LAT (points, $1 \sigma$ statistical error bars) and H.E.S.S. (grey shaded bow tie (Aharonian et al., 2008)) spectra from emission coincident with SNR CTB 37A. The dashed line, an extrapolation of the H.E.S.S. data into the Fermi-LAT energy range, clearly underpredicts the Fermi-LAT data. The discrepancy is somewhat ameliorated by including H.E.S.S. statistical errors (dotted lines) and is eliminated by very conservatively summing the H.E.S.S. statistical and systematic errors (dot-dashed line). The spectral tension remains after instead normalizing the best-fit H.E.S.S. hadron model to the Fermi-LAT data (solid curve). 


\section{Multiwavelength Spectrum}

By observing objects in multiple wavelengths, we are able to gain a more complete understanding of their internal workings, and SNRs are no exception. Figure 2 shows the gamma-ray spectrum for the Fermi-LAT source associated with CTB 37A.

\subsection{High Energy Gamma-rays}

The gamma-ray band is particularly sensitive to possible CR acceleration through the $\pi^{0}$ decay channel, initiated by the interaction of high energy hadrons, typically taken to be protons. In 2008, H.E.S.S. detected very high energy gamma-rays coincident with CTB 37A. That data, in combination with radio and X-ray analysis, suggested that a hadron-dominated emission scenario was more likely than a leptonic one, though the latter could not be excluded (Aharonian et al., 2008).

As the Fermi-LAT and H.E.S.S. energy ranges are complementary and the sources themselves coincident, we examined the extrapolation of the H.E.S.S. data into the Fermi-LAT range. The dashed line in Figure 2, showing this extrapolation, clearly underpredicts the Fermi-LAT measured spectrum. Including statistical errors somewhat ameliorates the discrepancy, though the global fit likelihood remains more than $8 \sigma$ worse than freely fitting the source. Very conservatively directly summing the statistical and systematic errors eliminates the difference, as seen from the dotted and dot-dashed lines, respectively.

If instead we use gtlike to fit the Fermi-LAT data with the H.E.S.S.indicated hadronic emission model (from $\pi^{0}$ decay) as seen in Figure 2 (solid curve), the Fermi-LAT data suggest that H.E.S.S. should observe more emission than it does. To generate the model, we used H.E.S.S. parameters for the $\mathrm{SNR}$ at $11.3 \mathrm{kpc}$ interacting with clouds of gas mass $\mathrm{N}_{\mathrm{H}}=6.7 \times 10^{4} \mathrm{M}_{\odot}$ with a power law index of $\gamma=2.30$ (Aharonian et al., 2008). While the discrepancy may arise from statistical or systematic errors or differences in source extension as the H.E.S.S. source is slightly smaller than and marginally offset from the Fermi-LAT source, it may also indicate two different spectral components. To determine if the latter is an actual possibility, we extended our model to include the radio and X-ray data.

\subsection{A Hadronic + Leptonic Model}

We fit a one-zone model using reasonable values and containing both hadronic ( $\pi^{0}$ decay) and leptonic (synchrotron, bremsstrahlung, and inverse 
Compton) emission to the multiwavelength data coincident with SNR CTB 37A, as shown in Figure 3.

We selected radio data tabulated by Kassim et al. (1991) with frequencies above $200 \mathrm{MHz}$, where absorption has a minimal effect, and fluxes both with errors and consistent with other measurements. For the $8800.0 \mathrm{MHz}$ band, we fixed the error at $10 \%$ of the total value, which is the same order of magnitude and slighlty larger than the errors in the other bands.

In a reasonable $20 \mu \mathrm{G}$ magnetic field ${ }^{4}$, leptons produce radio-band synchrotron emission. We calculated this leptonic model component according to the method of Ginzburg (1979) and Ghisellini et al. (1988) and assuming an electron population following a standard exponentially cutoff power law with a typical cutoff energy of $\mathrm{E}_{\text {cut }}=50 \mathrm{GeV}$. We fit the synchrotron component to the radio data, providing the lepton population spectral index and normalization of $\gamma_{\mathrm{e}}=-1.75$ and $\mathrm{N}_{\mathrm{e}}=3.66 \mathrm{e} / \mathrm{s} / \mathrm{cm}^{2} / \mathrm{GeV} / \mathrm{sr}(\sim 219$ times that of the local $\mathrm{CR}$ electron spectrum) at $1 \mathrm{GeV}$, respectively.

Leptons in this population may also interact with molecular clouds, producing bremsstrahlung emission proportional to the gas mass of the clouds in the region, estimated at $\mathrm{M}_{\mathrm{H}}=6.5 \times 10^{4} \mathrm{M}_{\odot}$ for the northern and central clouds of Reynoso and Mangum (2000), which are those most consistent with the Fermi-LAT emission. Leptons may also inverse Compton scatter off local starlight and the cosmic microwave background (e.g. as measured by WMAP), producing high energy photons. We calculated the inverse Compton emission using the method described by Blumenthal and Gould (1970) with optical and infrared interstellar radiation fields from Porter et al. (2008).

We combined the leptonic emission components with a hadronic model in which a high energy proton population, following a power law with index of -2.30 , interacts with ambient protons, producing $\pi^{0} \mathrm{~s}$ which decay to two photons, calculated following the prescription of Kamae et al. (2006). Using the same gas mass as for the bremsstrahlung component, we renormalized the hadronic gamma-ray flux to approximately fit the H.E.S.S. data, determining a $\mathrm{CR}$ proton enhancement factor of $\sim 11.6$ with respect to the local $\mathrm{CR}$ proton spectrum. The protons in this steady state model have a total energy

\footnotetext{
${ }^{4}$ Measurements of Zeeman splitting in $\mathrm{OH}$ masers coincident with CTB 37A provide an upper limit on the magnetic field magnitude of $1.5 \mathrm{mG}$ (Brogan et al., 2000). Such OH masers typically occur in molecular clouds carrying ambient magnetic fields which have been compressed by the SNR shock passage (Brogan et al., 2000). Thus, to obtain the value used, we scaled the field by the size of the cloud.
} 


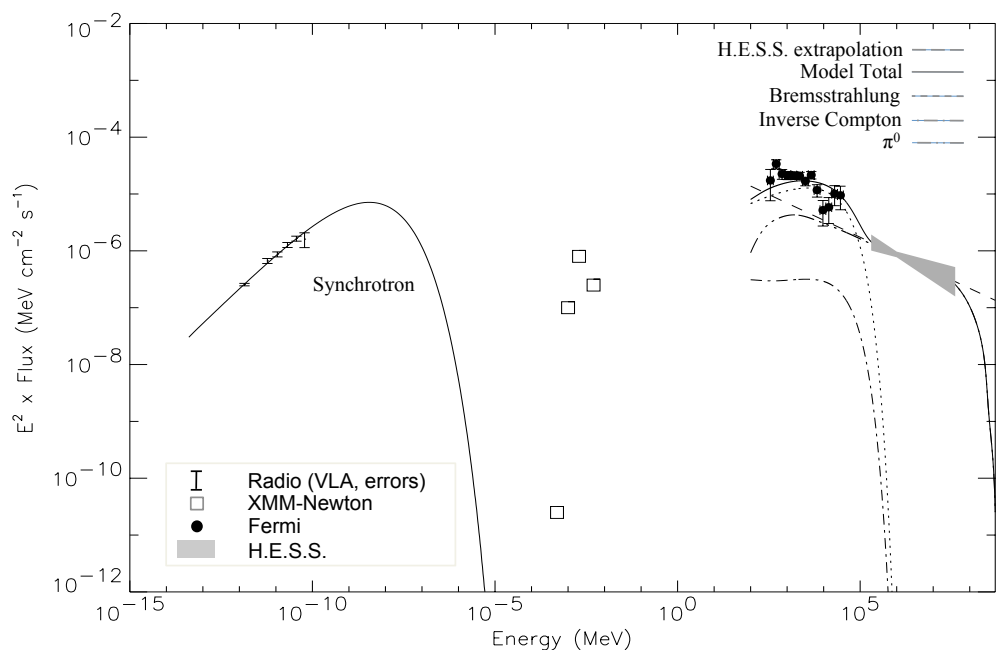

(a)

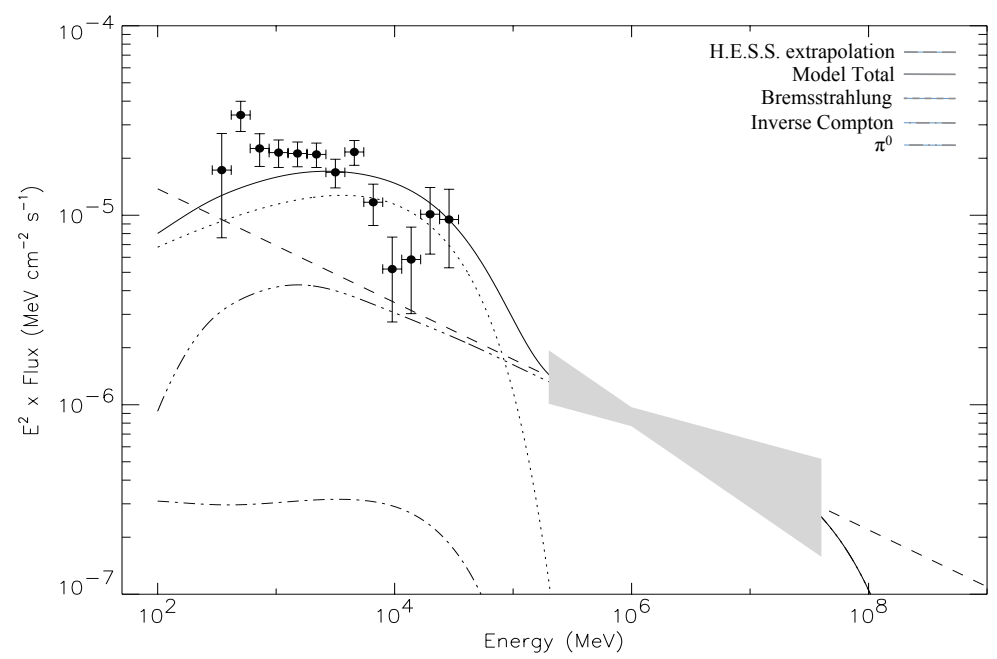

(b)

Figure 3: 3a. The multiwavelength spectrum of emission associated with CTB 37A, spanning from radio to very high energy gamma-ray emission. We fit the radio data (Kassim et al., 1991) with a synchrotron model (solid line) having a $20 \mu \mathrm{G}$ magnetic field, determining the lepton population's spectral index and normalization, having assumed a power law with exponential cutoff energy of $50 \mathrm{GeV}$. 3b. We then model the lepton population's bremsstrahlung (dashed line) and inverse Compton (dot-dashed line) emission. We adjusted the hadron population's pion emission (triple-dot-dashed line) from protonproton interaction in the same ambient clouds as used for the leptons to produce bremsstrahlung to approximately fit the H.E.S.S. data (grey bow tie) (Aharonian et al., 2008). The totall modeled emission (solid line at high energies) relatively well reproduces the Fermi-LAT data without further adjustments. The XMM-Newton X-ray data from the MOS1 and 2 and PN instruments (open squares) constrains the maximum synchrotron emission at the highest energies. 
of $\sim 1.2 \times 10^{50} \mathrm{ergs}$, implying a very reasonable $\sim 12 \%$ conversion efficiency for a canonical SN kinetic energy of $10^{51} \mathrm{ergs}$ and in good agreement with the H.E.S.S. prediction (Aharonian et al., 2008). The total lepton energy, at $\sim 5 \times 10^{49} \mathrm{ergs}$, is a reasonable roughly half the hadron energy. The proton energy corresponding to the maximum H.E.S.S. energy, $\mathrm{E}_{\mathrm{p} \text {,max }}$ between $10^{14} \mathrm{eV}$ and $10^{15} \mathrm{eV}$ (Aharonian et al., 2008), is commensurate with the maximum energy expected from SNe for particles of charge $Z=1$ (Brandt, 2010) and with the "knee" or change in CR spectral slope at $10^{15} \mathrm{eV}$.

Figure 3 shows the result of fitting this model to the multiwavelength data. The lepton population reproduces the radio-measured synchrotron emission while the combination of $\pi^{0}$ decay and bremsstrahlung emission reproduce the Fermi-LAT data, notably not previously fit, relatively well with no further adjustments. The inverse Compton component contributes at only about the $1 \%$ level. From this we anticipate obtaining similar values when performing a complete fit of all the data, allowing the magnetic field strength as well as the proton and electron populations' parameters $\left(\mathrm{N}_{\mathrm{p}}, \gamma_{\mathrm{p}}, \mathrm{N}_{\mathrm{e}}, \gamma_{\mathrm{e}}, \mathrm{E}_{\mathrm{e}, \text { cut }}\right)$ to vary.

As synchrotron emission can occur at wavelengths up to X-ray, we examined data from the XMM-Newton observatory. Using approximately $17 \mathrm{ks}$ of XMM-Newton observation from 1 March 2006 (ObsID 0306510101), we analyzed the data with the standard XMM-Newton Science Analysis Software (SAS v.9.0) $)^{5}$. We extracted the spectral flux from the region of excess X-ray flux coincident with the Fermi-LAT source. H.E.S.S. analysis of the same data and of Chandra data in the same region found the spectra to be compatible with absorbed thermal emission (Aharonian et al., 2008). Our XMM-Newton analysis yields a flux likewise consistent with an absorbed thermal spectrum with the same order parameters as those given for the Chandra data: a temperature of several hundred $\mathrm{eV}$ and column density of a few $\times 10^{22} \mathrm{~cm}^{-2}$. We suspect we slightly underestimated the parameters relative to those found for Chandra as we extracted the spectrum under the point source assumption.

Assuming the X-ray emission is thermal, the (lowest energy) x-ray data thus constrains the highest energy synchrotron emission the lepton population may produce: for the reasonable model tested herein, a cutoff energy greater than $\sim 1.5 \mathrm{TeV}$ would produce an excess of synchrotron emission

\footnotetext{
${ }^{5}$ Available at http://xmm.esac.esa.int/xsa.
} 
not observed in X-rays. In a similar fashion, for this model and fixed set of parameters, we can constrain $\mathrm{E}_{\mathrm{e}, \text { cut }} \lesssim 50 \mathrm{GeV}$ to avoid excessive flux from bremsstrahlung emission in the H.E.S.S. domain. We anticipate similar constraints from performing a full fit of all the parameters.

\section{Conclusions}

We robustly detect a source coincident with SNR CTB 37A at $18.6 \sigma$ using the Fermi-LAT. Fermi-LAT $\gamma$-ray data, in concert with radio, X-ray, and $\mathrm{TeV}$ data, allows us to determine the type of emission and its characteristics by determining the most likely model. In this work, we are able to fit the multiwavelength emission with a reasonable model combining emission from hadronic and leptonic populations. Thus, SNR CTB 37A is a potential CR accelerator.

We are currently finishing a complete fit of the multiwavelength data over the allowable ranges for the local magnetic field and the leptonic and hadronic populations' parameters to determine their most probable values. In particular, this method permits us to robustly determine whether one population's emission dominates over the other or if both are necessary to reproduce the observed data. Since our initial model with reasonable values reproduced the Fermi-LAT data moderately well, we anticipate similar values for the more robust fit.

Additional sources of data, such as microwave (e.g. Planck), infrared (e.g. Spitzer), and optical (which we will obtain when the source reemerges from behind bright solar system objects) data may further help disentangle the emission models. By assembling individual CR source candidates such as SNRs into statistically significant populations, we will improve our understanding of the potential source classes, allowing comparison to properties derived from direct $\mathrm{CR}$ detection experiments, and more fully illuminating a hundred-year mystery.

\section{Acknowledgements}

The Fermi LAT Collaboration acknowledges generous ongoing support from a number of agencies and institutes that have supported both the development and the operation of the LAT as well as scientific data analysis. These include the National Aeronautics and Space Administration and the Department of Energy in the United States, the Commissariat à l'Energie 
Atomique and the Centre National de la Recherche Scientifique / Institut National de Physique Nucléaire et de Physique des Particules in France, the Agenzia Spaziale Italiana and the Istituto Nazionale di Fisica Nucleare in Italy, the Ministry of Education, Culture, Sports, Science and Technology (MEXT), High Energy Accelerator Research Organization (KEK) and Japan Aerospace Exploration Agency (JAXA) in Japan, and the K. A. Wallenberg Foundation, the Swedish Research Council and the Swedish National Space Board in Sweden.

Additional support for science analysis during the operations phase is gratefully acknowledged from the Istituto Nazionale di Astrofisica in Italy and the Centre National d'Études Spatiales in France.

\section{References}

Abdo, A. A., Ackermann, M., Ajello, M., et al. Jun. 2010a. Fermi Large Area Telescope First Source Catalog. ApJS 188, 405-436.

Abdo, A. A., Ackermann, M., Ajello, M., et al. Apr. 2010b. The First Fermi Large Area Telescope Catalog of Gamma-ray Pulsars. ApJS 187, 460-494.

Abdo, A. A., Ackermann, M., Ajello, M., et al. Mar. 2010c. Observations of the Large Magellanic Cloud with Fermi. AAP 512, A7+.

Abdo, A. A., Ackermann, M., Ajello, M., et al. Mar. 2010d. Observation of Supernova Remnant IC 443 with the Fermi Large Area Telescope. ApJ 712, 459-468.

Aharonian, F., Akhperjanian, A. G., Barres de Almeida, U., et al. Nov. 2008. Discovery of a VHE gamma-ray source coincident with the supernova remnant CTB 37A. AAP 490, 685-693.

Atwood, W. B., Abdo, A. A., Ackermann, M., et al. Jun. 2009. The Large Area Telescope on the Fermi Gamma-Ray Space Telescope Mission. ApJ 697, 1071-1102.

Blumenthal, G. R., Gould, R. J., 1970. Bremsstrahlung, Synchrotron Radiation, and Compton Scattering of High-Energy Electrons Traversing Dilute Gases. Reviews of Modern Physics 42, 237-271.

Brandt, T. J., 2010. On high energy cosmic rays from the CREAM instrument. Ph.D. thesis, The Ohio State University. 
Brogan, C. L., Frail, D. A., Goss, W. M., Troland, T. H., Jul. 2000. OH Zeeman Magnetic Field Detections toward Five Supernova Remnants Using the VLA. ApJ 537, 875-890.

Castro, D. and Slane, P., Jul. 2010. Fermi Large Area Telescope Observations of Supernova Remnants Interacting with Molecular Clouds. ApJ 717, 372378.

Dormody, M., et al., 2011. In preparation.

Frail, D. A., Goss, W. M., Reynoso, E. M., Giacani, E. B., Green, A. J., Otrupcek, R., Apr. 1996. A Survey for OH (1720 MHz) Maser Emission Toward Supernova Remnants. AJ 111, 1651-+.

Ghisellini, G., Guilbert, P. W., Svensson, R., Nov. 1988. The synchrotron boiler. ApJL 334, L5-L8.

Ginzburg, V. L., 1979. Theoretical physics and astrophysics. International Series in Natural Philosophy, Oxford: Pergamon.

Green, D. A., Mar. 2009. A revised Galactic supernova remnant catalogue. Bulletin of the Astronomical Society of India 37, 45-+, available at http: //www.mrao.cam.ac.uk/surveys/snrs/.

Kamae, T., Karlsson, N., Mizuno, T., Abe, T., Koi, T., Aug. 2006. Parameterization of $\gamma, e^{+/-}$, and Neutrino Spectra Produced by p-p Interaction in Astronomical Environments. ApJ 647, 692-708.

Kassim, N. E., Weiler, K. W., Baum, S. A., Jun. 1991. A new look at the 'jet' in the CTB 37A/B supernova remnant complex. ApJ 374, 212-217.

Porter, T. A., Moskalenko, I. V., Strong, A. W., Orlando, E., Bouchet, L., Jul. 2008. Inverse Compton Origin of the Hard X-Ray and Soft GammaRay Emission from the Galactic Ridge. ApJ 682, 400-407.

Reynoso, E. M., Mangum, J. G., Dec. 2000. CO Observations toward Supernova Remnants with Associated OH 1720 MHZ Masers. ApJ 545, 874-884. 